\title{
Significant of BER Detection Using Hybrid Genetic Algorithm for CDMA System
}

\author{
Anwar Hassan Ibrahim \\ Electrical Engineering Department, Qassim University, Qassim, Saudi Arabia \\ Email: dr.anwar@qec.edu.sa
}

How to cite this paper: Ibrahim, A.H. (2018) Significant of BER Detection Using Hybrid Genetic Algorithm for CDMA System. Journal of Computer and Communications, 6, 31-39. https://doi.org/10.4236/jcc.2018.65003

Received: April 13, 2018

Accepted: May 19, 2018

Published: May 22, 2018

Copyright $\odot 2018$ by author and Scientific Research Publishing Inc. This work is licensed under the Creative Commons Attribution International License (CC BY 4.0).

http://creativecommons.org/licenses/by/4.0/

\begin{abstract}
In this paper, CDMA system is considered to enhance the detection techniques, which uses genetic algorithm practice to introduce an optimum multi-user indicator based on bit synchronization for code division multiple access via high level of noise (Gaussian channel). The proposed method presented in this paper is to define the transmitted bit sequence and optimization in the receiver using hybrid GA scheme, which considered a complicated method to match the users' bits correlation metric. A Matlab simulation results shows that, the BER performance under certain condition of SNR for multi-user detector methods indicate the single-user convolution bound, despite the fact that a lower complication as associated to the best multi-user detection. Particularly the hybrid GA structure is more suitable for a high number of users and suitable for CDMA system enhancement in order to perform lower computational complexity in shorter time period.
\end{abstract}

\section{Keywords \\ CDMA, GA, BER, SNR}

\section{Introduction}

CDMA system is one of various methods developed for signal multiplexing that has been taken an important role in cellular and personal communication system [1] [2]. The optimal multi-user detector [3] maximizes the combined subsequent probability with a complex number of users that grows exponentially. The high computational convolution involvement has determined for suboptimal multi-user detectors through much process concentrated in computational complexity.

The proposed multi-user detection technique developed to utilize the genetic algorithm in contrast to previous approaches. Furthermore, genetic algorithm is 
not hybridized with any detector other than a conventional detector at initial stage [4]. The procedure of this algorithm significantly decreases the number of tasks as well as the entire cost of the planned detector. Several number of methods implemented DS-CDMA system for user data verification and error detection were observed, but none of them realized the genetic algorithm performance for bit error rate significant and accuracy. A Matlab simulation shows the proposed genetic algorithm at the receiver recognition performance close to the optimal detector, as defined by the bit-error-rate (BER). The Possible of the genetic algorithm detector consider a suboptimal direct sequence for multi-user detector is significantly established.

CDMA/GA implemented using Matlab simulation to allow various code detectors with various users. The goal is to measure the performance of the detector and allow them for multi-detector to be tested. The main criteria used to estimate the performance of genetic algorithm in order to reach the maximum possibility detection by using the fitness value and probability to select the population at each generation and recombination genetic algorithm.

To study the CDMA using system performance, a software model of the system was developed. The model was implemented using Matlab program because it was constructed with multi functions flexible to estimate the bit error rate versus SNR.

\section{Bit Error Rate and SNR}

An important application of spread spectrum (SS) systems is a multiple-access communications system, which means several users, may possibly access to a shared channel and communicate with others.

The Probability of Error performance in the receiver part is presented and found that, the analyses accept the correlated noise between received antenna array fundamentals, and random number of self-determining user proposed. The antenna array and effective environment impairments, such as random noise generation and additive white Gaussian Noise, on overall space-path range that can be directly assessed. Moreover, it makes the usual assumption that power control is used to enable all users' transmissions that reached the user of interest with the same power. Under these conditions, it can be shown, that the receiver bit error probability can be approximated. The Equations (1)-(3) define probability of error and signal to noise ratio [5].

$$
P_{E}=Q(\sqrt{S N R})
$$

where

$$
\begin{gathered}
P_{E}=Q\left(\sqrt{\frac{A^{2} T b}{N o}}\right)=Q\left(\sqrt{\frac{2 E b}{N o}}\right) \\
S N R=\left(\frac{K-1}{3 N}+\frac{N o}{2 E b}\right)^{-1}
\end{gathered}
$$


in which $K$ is the number of users and $N$ is the number of chips per bit, or the processing gain.

\section{Genetic Operators}

The selection of genetic algorithm is an operator based on the fitness value and fittest chromosome. The concept of this algorithm is to match the existence of the fittest mechanism in nature condition. Most generally the relation of the matrix shows in Figure 1 expressed how crossover and mutation occur.

The mutation operator basically changes each bit in the string randomly through a user measured probability. In different to the crossover, means that the probability of the change is set to a small, such as 0.01 . Bellow is an example of the change of the fourth bit of the second off spring $1111-1 \quad \begin{array}{llllll}1 & 1 & -1 & 1 & \text { (muta- }\end{array}$ tion point), after the mutation it become $11111111-11$.

\subsection{Fitness Function}

The most important factor of the fitness function is to estimate the position of every chromosome. The order of the fitness function is essential to be no-negative. In the user detection, the objective of this process is to maximize of the cost of the function.

$$
C(b)=2 y^{1} b-b^{1} H b
$$

The objective function is not mandatory to be positive in all situations. For this reason it is required to plan the objective value in accordance to the fitness value. The following objective is plan to fitness change is used [5]

$$
F(b)=K+(C(b)-c w)
$$

At this time, the objective rate of the worst case in chromosome of the current population considered to be positive constant.

\subsection{Setting Genetic Algorithm}

There are no all-inclusive and standard parameter settings for GA, which implies that to discover a source that refers to any such settings. Rather, used a concept for simple discover a few papers, which will help to adjust the parameters for the specific value needed, and find many, numerous others that basically take a "standard" arrangement of parameter settings without much endeavor to legitimize them.

The good method for the optimal setting of genetic algorithm parameters is significantly depending on the problem. The main parameters to be set according to [6] were:

\begin{tabular}{rlllllllll}
\hline \multicolumn{1}{|c|}{ Parent's } & \multicolumn{1}{c|}{ crossover spring } \\
-1 & 1 & 1 & -1 & 1 & 1 & -1 & 1 \\
1 & 1 & 1 & 1 & -1 & -1 & -1 & -1
\end{tabular}

Figure 1. Crossover and mutation. 
1) Population size (NP);

2) Replacement strategy;

3) Crossover probability (Pc);

4) Mutation probability (Pm).

The time required to attempt each parent possibility and select the most way to perform the best genetic. Nevertheless, the best choice adoptions are not unusually in many GA operations to contrast the population size from a small value to a reasonable number. In this paper the concept employ the crossover in off spring operator with close to 1 and changing operator to entirely bits with close to 0 using wheel selection method for regular replacement.

\subsection{Implementation of GA Based Multi-User Detection}

The target of genetic algorithm implementation initiates with a population of random chromosomes, to represent a binary strings. The execution and mutation operator of the population size and chromosomes length were complicated due to dependent problem. Accordingly the members of the population are frequently strings of symbols that signify the conceivable solutions to the problem to be resolved. Every associated member of the population at each group is estimated, and signified to the fully fitness value. The assigned target based on a probability to be designated for reproduction. Subsequently the selection of crossover for genetic is applied to pairs of parents in order to create the off springs, which will be controlled by mutation operator and the implantation into a different population creating the subsequent generation individuals [7] [8].

The goal of crossover operators is to find completely data structure for the point for two selected members of population and associations with their respective chromosomes to generate the off springs during crossover. The mutation operator of the initial size throughout selection increases the efficiency of the algorithm. Based on the mutation probability the input restriction chooses the fitting value for individuals first. The requirement of member selection from the population will change some portions of it chromosomes. In order to assess the algorithm for multiple user detection assumed that all members of the population with worst fitness values were exchanged with the new individuals and obtained objective function accordingly.

\section{Proposed Genetic Algorithm Process}

The Genetic algorithm play very important role to achieve its work in short duration of many schemes of our life. Based on crossover construction probability for the input parameter a collection of individual's chromosome calculated on a simplex type of population according to crossover operation as shown in the simple flow chart in Figure 2.

The mutation prospect is related to the fitness criteria and selection as the operation is not applied to the new population. The best individuals is depend on mutation and crossover by complete labeled as same as natural biological progress. 


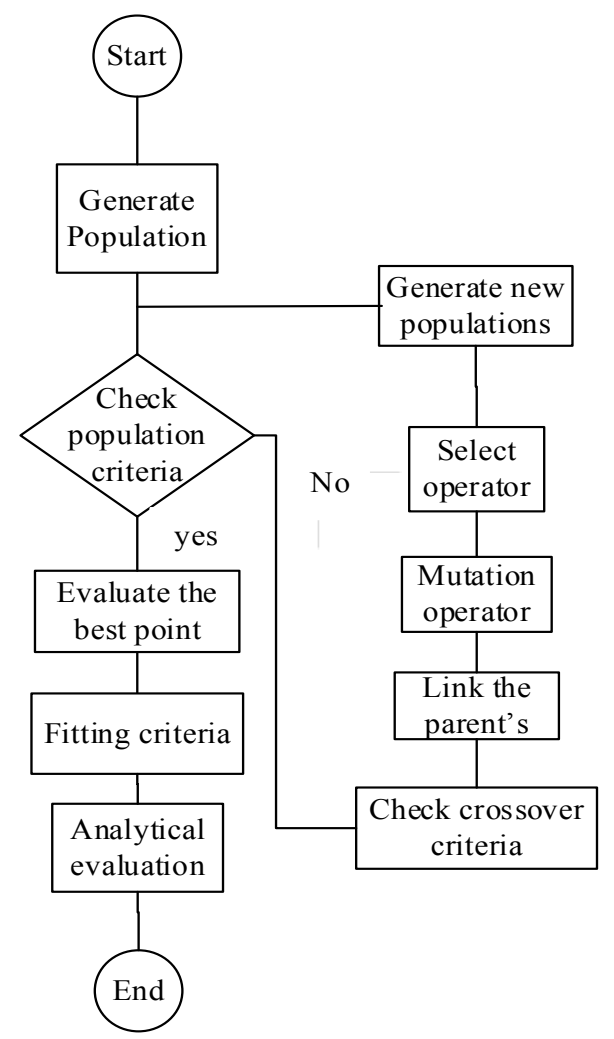

Figure 2. Genetic algorithm process.

GAs operates on potential solution of mutation of population through applying the principle of fitting criteria to produce better estimation. For the process of selecting individuals from natural genetics is based on the level of fitness and mutation. This process indicates that the progress of populations was better matched to their setting to compute the optimum point.

\section{Detector Performance}

The bit error probability is generally considered a desirable performance level in multi-user detection in communications. However, the complex mode of the bit error rate (BER) function prevents from developing an optimal solution for the mutli-user detection weights for single user detection. In the situation of the ideal detector for whichever synchronous or asynchronous communication, the probability of error is very complex and uninteresting for estimation in this case.

The ideal bit error rate usually evaluated through the conventional and the de-correlating detector [9]. The parameters used for the simulations are based on population criteria which evaluation the fitting conditions for the detectors to complete the properties analyzed. Since the fitting criteria is not match to crossover criteria, the mutation operator will requires the parent algorithm and the knowledge of the channel population with the average of the error function calculated. Moreover, the optimal of the step size and analytical evaluation of the algorithm allows constituted by the maximum affordable number of generations in term of population. 


\section{Results}

The simulation analysis in results explains the Bit error rate and signal to noise ratio preparation complexity. Matlab software evaluates the condition of multi-user detectors via multi procedures described according to the optimization of genetic algorithm criteria. The purposes of this simulation are to study the BER performance as shown in Figures 3-6. CDMA systems running over a pseudo-noise code ( $\mathrm{PN}$ code) channel shows a significant challenge in the population criteria decision according to the fitting criteria with randomly designated with long spreading factor codes are used. Assume that, all users are transmitting with the equal power.

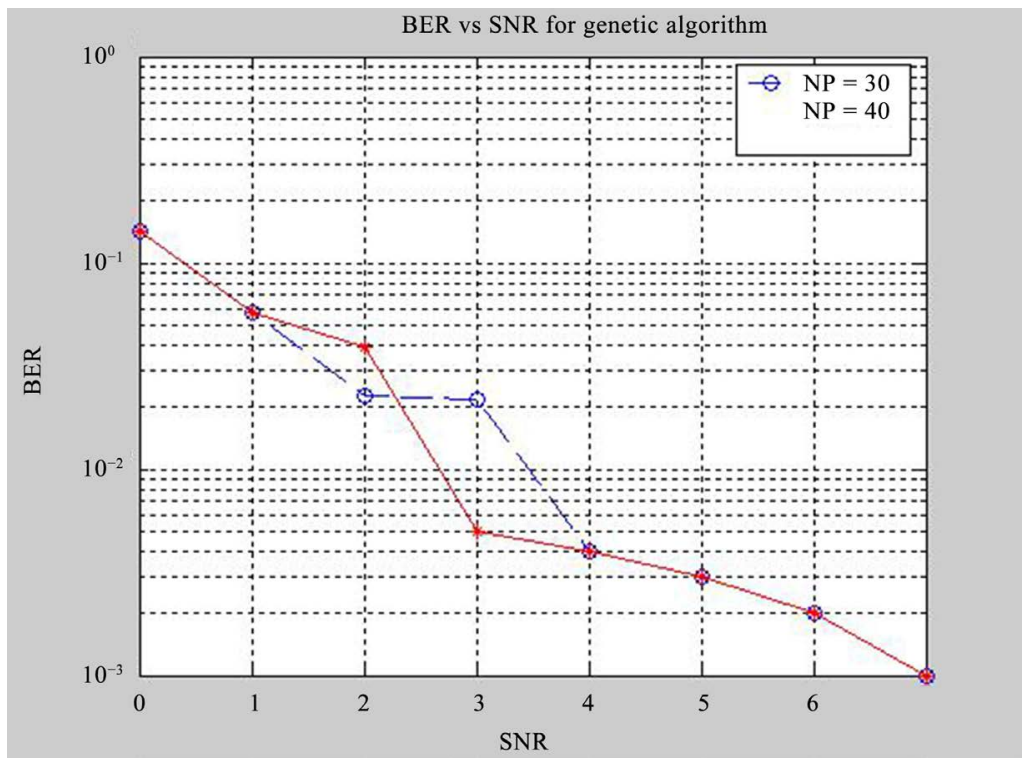

Figure 3. User distribution for given BER.

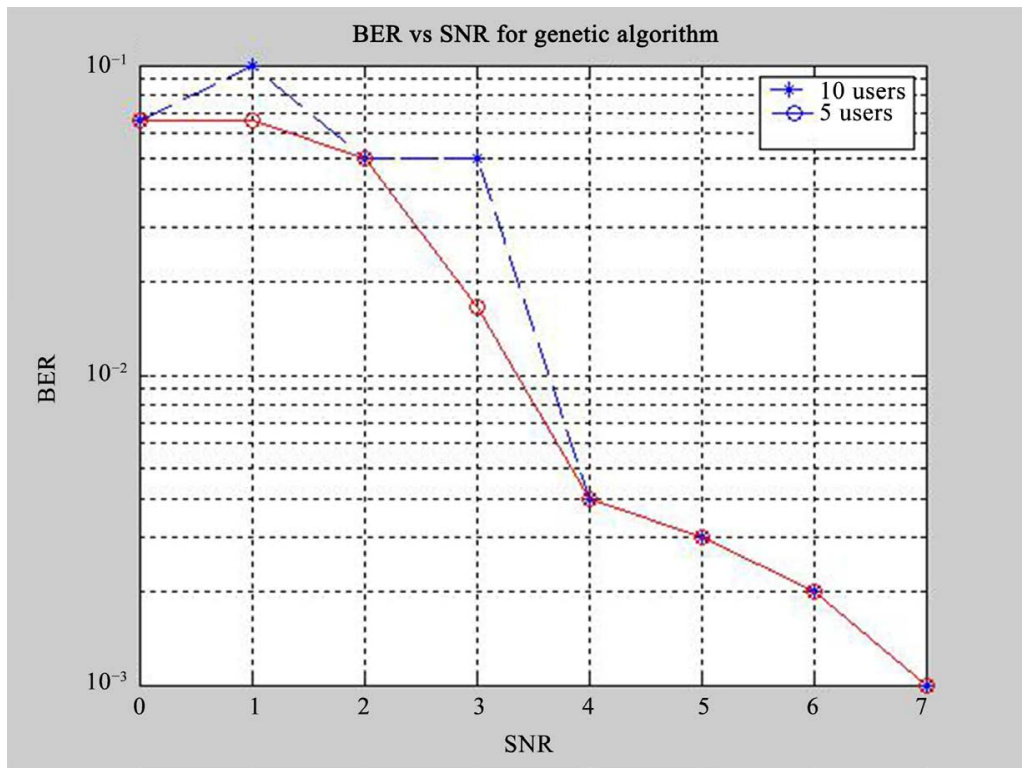

Figure 4. BER vs SNR (Pop size $=80)$. 


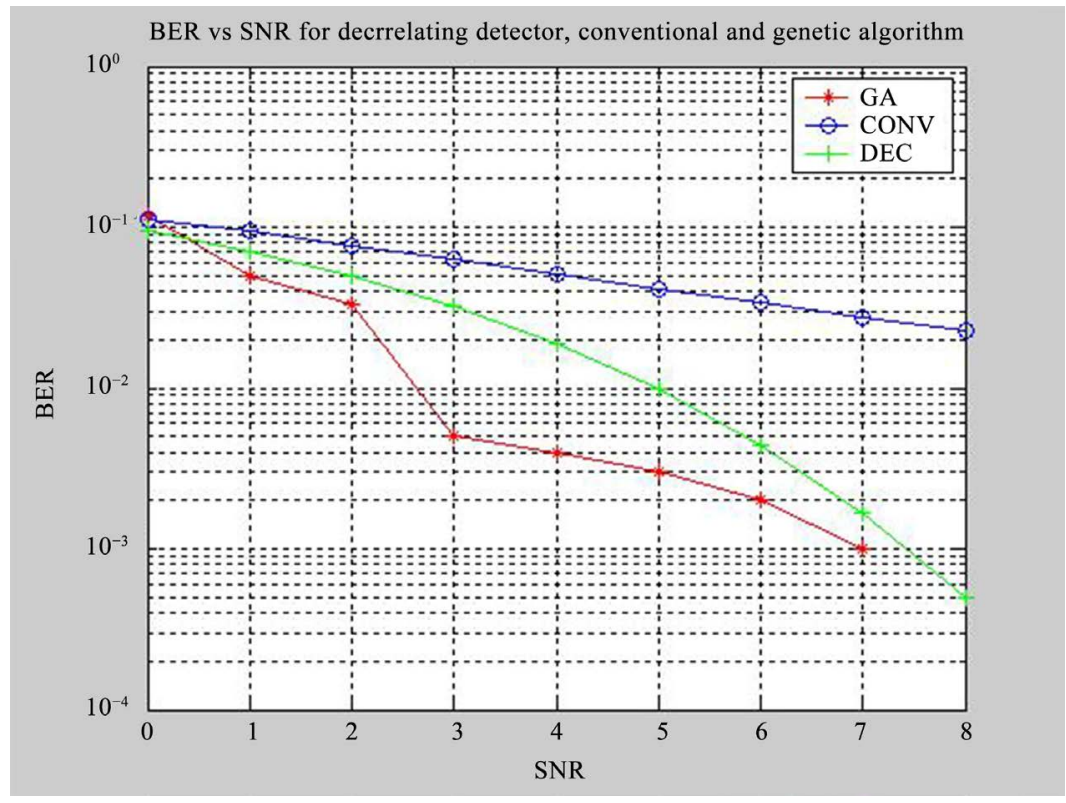

Figure 5. BER vs SNR (Pop size $=40)$.

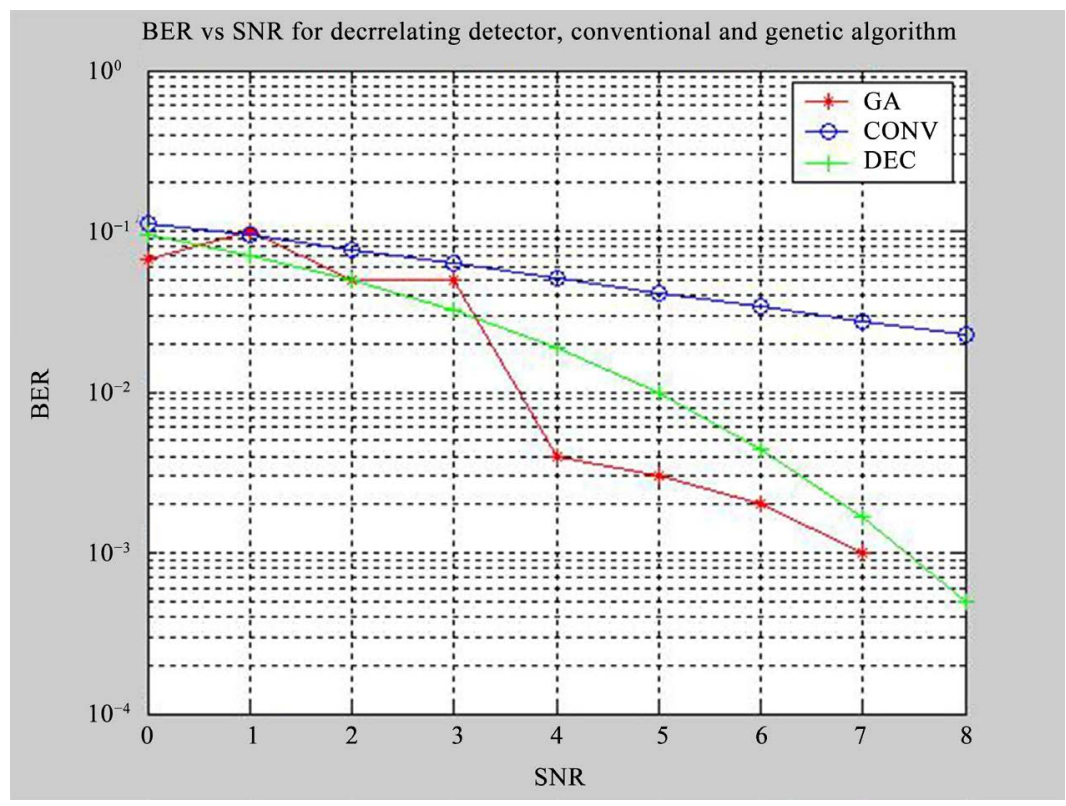

Figure 6. BER vs SNR (Pop size $=60)$.

This figure illustrates the BER vs SNR for genetic algorithm system under distributed of the population size. When the number of user is constant $=5$.

In this figure the result showed the BER vs SNR for genetic algorithm system under distributed users. That means the number of user is changing when the size of population is constant $=80$, under 5 and 10 users condition.

Figure 5 clarify shows a comparison between genetic algorithm, conventional and de-correlation detector for 10 users and 40 population size.

Figure 6 explains comparison between GA, conventional and de-correlation detector for 10 users and population size $=60$, regarding to BER vs SNR. 
The simulation activates performed multi-user in order to compute the exact probability of error performance versus signal to noise ratio by spreading sequences gold codes of length 31 . All results presented in this paper are targeted towards the study of the system performance in term of bit error rate (BER) for (SNR) ranging from $0 \mathrm{~dB}$ to $8 \mathrm{~dB}$.

The work was done using conventional, de-correlating detectors compared with genetic algorithm detector which based on multi-user detector for sharing and improving the optimum multi-user detection. In the first case from the Figure 1 and Figure 2, the simulation was applied for various users and population size. It is understandable that the BER performance of GA. The error decreases gradually by using fitness function for GA. From the results of the Figure 3 and Figure 4, the simulation has shown that the conventional detector (CD) is significantly superior in performance, but with increased computational complexity. Genetic algorithm (GA) viewed as an optimum detector that gives better performance.

\section{Conclusions}

The simulation results have shown that the genetic algorithm detector is an efficient and effective method for multi-user detection. The change in the values of the crossover and mutation parameters for GA can affect the output results. The probability of mutation (PM) versus to crossover is normally very small. It is significantly marked as shown in the figures, that the performance is improved as we increase the population size (NP) and the performance is poor when NP is too small with genetic algorithm reaching the optimum performance when NP is reached 40 or above.

The results can be better when the number of iteration ( $S$ ) is increased because the processes take a long duration to achieve this work.

\section{References}

[1] Viterbi, A.J. (1994) The Orthogonal-Random Waveform Dichotomy for Digital Mobile Personal Communication. IEEE Personal Communications, 1, 18-24.

[2] Kohno, R., Median, R. and Milstein, L.B. (1991) Spread Spectrum Access Methods for Wireless Communications. IEEE Communications Magazine, 33, 58-67.

[3] Kechriotis, G.I. and Manolakos, E.S. (1996) Hopfield Neural Network Implementation of the Optimal CDMA Multiuser Detector. IEEE Transactions on Neural Net works, 7, 131-141. https://doi.org/10.1109/72.478397

[4] Ergun, C. and Hacioglu, K. (1998) Application of a Genetic Algorithm to Multi-Stage Detection in CDMA Systems. Electrotechnical Conference, 1998, MELECON 98, 9 th Mediterranean, Tel-Aviv, 18-20 May 1998, 846-850.

[5] Alias, M.Y., Chen, L. and Hanzo, L. (2004) Genetic Algorithm Assisted Minimum Bit Error Rate Multiuser Detection in Multiple Antenna Aided OFDM. 2004 IEEE 60th Vehicular Technology Conference, VTC2004-Fall, Los Angeles, 26-29 September 2004, 548-552.

[6] Ziemer, R.E. and Tranter, W.H. (2002) Principles of Communications Systems Modulation and Noise. 5th Edition, Wiley, Hoboken, 436-440. 
[7] Ergun, C. and Hacioglu, K. (2000) Multiuser Detection Using a Genetic Algorithm in CDMA Communications Systems. IEEE Transactions on Communications, 48, 1374-1383. https://doi.org/10.1109/26.864174

[8] Aporntewan, C. and Chongstitvatana, P. (2011) A Hardware Implementation of the Compact Genetic Algorithm. Proceedings of the 2001 Congress on Evolutionary Computation, Seoul, 27-30 May 2001, 624-629.

[9] Tang, K.S., Man, K.F., Kwong, S. and He, Q. (1996) Genetic Algorithms and Their Applications. IEEE Signal Processing Magazine, 13, 22-37.

https://doi.org/10.1109/79.543973 